\section{Endovascular treatment experience in acute ischemic stroke}

\author{
Hacı Ali Erdoğan ${ }^{1}$, İbrahim Acır ${ }^{2 *}$ and Vildan Yayla ${ }^{1}$ \\ 'Bakırköy Dr. Sadi Konuk Training and Research Hospital Istanbul, Turkey \\ ${ }^{2}$ Başakşehir Çam and Sakura City Hospital Istanbul, Turkey
}

\section{Abstract}

Background and Objective: Thrombolytic and mechanical thrombectomy therapies are proven treatment methods in patients with acute stroke. Aim is to share our experience in acute stroke therapy with colleagues.

Material and methods: In this study we evaluated the patients who underwent MT or MT + IV-tPA between 2018-2019 retrospectively. Demographic features, comorbid diseases of patients, symptom onset-to-gate and symptom gate-to-puncture durations, mRS (Modified Rankin Score) and NIHSS (National Institutes of Health Stroke Scale) score, treatment method and degree of recanalization were listed.

Results: MT was applied to 29 patients, MT + bolus IV-tPA was applied to 12 patients and MT + full dose IV-tPA was applied to 7 patients. The mean age was $66 \pm 15$ years, arrival mRS was $2 \pm 2$, arrival NIHSS score was $14 \pm 5$, onset-to-gate duration was 185 minutes and gate-topuncture duration was 118 minutes.

Conclusion: The rate of recanalization, functional independence and mortality were similar to the HERMES study. It was observed a higher rate of intracranial hemorrhage in patients who received bolus or full dose IV-Tpa compared to patients who underwent MT. These results have led us to question the necessity of giving bolus or full dose IV-tPA before MT. Onset-to-gate and gate-to-puncture durations were found longer than the recommended durations. Rapid and effective management of AIS patients will provide good clinical results.

\section{More Information}

*Address for Correspondence: İbrahim Acır Başakşehir Çam and Sakura City Hospital Istanbul, Turkey, Tel: +905444498678; Email: iacir33@gmail.com

Submitted: April 19, 2021 Approved: April 23, 2021 Published: April 26, 2021

How to cite this article: Erdoğan $\mathrm{HA}, \mathrm{Acır} \mathrm{I}$, Yayla V. Endovascular treatment experience in acute ischemic stroke. J Neurosci Neurol Disord. 2021; 5: 026-028.

DOI: 10.29328/journal.jnnd.1001047

ORCiD: orcid.org/0000-0002-9650-8022

Copyright: @ 2021 Erdoğan HA, et al. This is an open access article distributed under the Creative Commons Attribution License, which permits unrestricted use, distribution, and reproduction in any medium, provided the original work is properly cited.

Keywords: Acute stroke; Endovascular treatment

Check for updates

OPEN ACCESS

\section{Introduction}

Stroke is one of the important diseases in the world that can cause disability. In the hyperacute period, the application of mechanical thrombectomy with retractable stents in major vascular occlusions is among the proven method of treatment. According to the recent studies, mechanical thrombectomy procedure, which is recommended to be applied with iv-tPA within 4.5 hours, can be applied in suitable patients for up to 24 hours. The goal in treatment is complete recanalization (TICI 2b/3) [1]. General data and parameters of patients who underwent mechanical thrombectomy (MT) or MT + IV-tPA in our stroke center in the last 2 years were presented.

\section{Material and methods}

Patients who underwent MT or MT + IV-tPA between 20182019 at our stroke center were evaluated retrospectively. Demographic features and comorbid diseases of patients were listed. Symptom onset-to-gate and gate-to-puncture durations, arrival-exit mRS (Modified Rankin Score) and
NIHSS (National Institutes of Health Stroke Scale) score, treatment method and degree of recanalization were recorded. And the relationship between these datas and prognosis was evaluated. Descriptive statistics are used to define continuous variables. Mann Whitney $U$ test was used for a comparison of two variables that are not suitable for independent distribution and normal distribution. Chi-Square (or Fisher Exact test where appropriate) was used to examine the relationship between categorical variables. The statistical significance level was described as 0.05 . Analyzes were made using the MedCalc Statistical Software version 12.7.7 Program.

\section{Results}

The number of patients who underwent MT or MT + IV-tPA was 48 (27 male/21 female). MT was applied to 29 patients, MT + bolus IV-tPA was applied to 12 patients and MT + full dose IV-tPA was applied to 7 patients. Hypertension (HT), atrial fibrillation (AF) and diabetes mellitus (DM) were the most common comorbid diseases (respectively 48\%, 23\% and $15 \%$ ). The mean age was $66 \pm 15$ years, arrival mRS was 
$2 \pm 2$, arrival NIHSS score was $14 \pm 5$, onset-to-gate duration was 185 minutes and gate-to-puncture duration was 118 minutes (Table 1). Complete or partial recanalization was achieved in the majority of patients undergoing MT or MT + IV-tPA $(64 \%)$. The $24^{\text {th }}$ hour NIHSS score was calculated as 12.5 on average, and the $24^{\text {th }}$ hour mRS was 4 on average. Hemorrhagic transformation occurred in $73 \%$ of patients. Five of the patients who underwent thrombectomy died.

\section{Discussion}

According to the World Health Organization (WHO), stroke is a clinical syndrome characterized by signs and symptoms of cerebral function loss. In acute ischemic stroke (AIS), IV-tPA and MT are proven treatment methods for best clinical outcomes. Intravenous thrombolytic therapy with recombinant tissue plasminogen activator (rt-PA) is a proven method of treatment in patients presenting within the first four and a half hours with acute ischemic stroke [2,3]. In the AHA 2015 (American Heart Association 2015) guideline, endovascular treatment with stent-retriever in selected patients is class IA recommendation [4]. In addition, in DAWN and DEFUSE 3 studies, it has been shown that endovascular treatment may be beneficial in patients with proximal artery occlusion up to the 16-24 hours [5,6]. In HERMES (Highly Effective Reperfusion Evaluated in Multiple Endovascular Stroke) meta-analysis, the recanalization rate was $71 \%$, while in another meta-analysis this rate was reported as $81 \%[7,8]$. Complete recanalization (TICI2b/3) was achieved in our patient group at a rate of $64 \%$. Functional independence was calculated as $19.5 \%$ and our mortality rate was around $10 \%$. Our study is similar to the HERMES study in this regard. On the other hand, our study covers the short term, functional improvement after 90 days could not be evaluated.

In the DEFUSE study, the $90^{\text {th }}$ day mRS and NIHSS scores of patients who underwent MT + ivTPA were found significantly lower, and there was no significant difference in the number of patients who developed hemorrhagic transformation [6]. In

\begin{tabular}{|c|c|c|c|}
\hline & MT + IV-tPA & MT & \multirow{3}{*}{$p$} \\
\hline & Mean + SD & Mean + SD & \\
\hline & Med. (Min.-Max.) & Med. (Min.-Max.) & \\
\hline \multirow{2}{*}{ AGE } & $66 \pm 14$ & $67 \pm 16$ & \multirow{2}{*}{0,941} \\
\hline & $65(45-89)$ & $70(32-86)$ & \\
\hline \multirow{2}{*}{ ARRIVAL NIHSS } & $16 \pm 4$ & $14 \pm 6$ & \multirow{2}{*}{0,184} \\
\hline & $15(10-24)$ & $13(2-25)$ & \\
\hline \multirow{2}{*}{ ARRIVAL mRS } & $2 \pm 2$ & $2 \pm 2$ & \multirow{2}{*}{0,724} \\
\hline & $1(0-5)$ & $1(0-5)$ & \\
\hline \multirow{2}{*}{ ONSET-TO-GATE (min.) } & $136 \pm 84$ & $218 \pm 219$ & \multirow{2}{*}{0,512} \\
\hline & $120(30-270)$ & $150(15-780)$ & \\
\hline \multirow{2}{*}{ GATE-TO-PUNCTURE (min.) } & $82 \pm 64$ & $142 \pm 255$ & \multirow{2}{*}{0,15} \\
\hline & $60(30-270)$ & $90(30-1440)$ & \\
\hline \multirow{2}{*}{ DISCHARGE NIHSS } & $17 \pm 9$ & $12 \pm 8$ & \multirow{2}{*}{0,029} \\
\hline & $16(0-30)$ & $10(0-30)$ & \\
\hline \multirow{2}{*}{ DISCHARGE mRS } & $4 \pm 2$ & $4 \pm 2$ & \multirow{2}{*}{0,077} \\
\hline & $5(0-6)$ & $4(0-6)$ & \\
\hline
\end{tabular}

SD: Standart Deviation; Min: Minutes our study, MT was applied to 29 patients, MT + bolus IV-tPA applied to 12 patients and MT + full dose IV-tPA was applied to 7 patients. Intracranial hemorrhage was found in $65 \%$ of patients who underwent MT, $83 \%$ of patients who underwent MT + bolus IV-tPA, and $85 \%$ of patients who underwent $\mathrm{MT}+$ full dose IV-tPA. According to our data, there was no significant difference in terms of the risk of intracranial hemorrhage in patients who received bolus or full dose IVTpa, but it was observed at a higher rate compared to patients who underwent MT. These results have led us to question the necessity of giving bolus or full dose IV-tPA before MT.

Inourstudy, $\mathrm{HT}, \mathrm{AF}$ and DMwerethemostcommon comorbid diseases in patients who underwent MT or IV-tPA + MT. Intracranial hemorrhage revealed in $82 \%$ of patients with HT, $62 \%$ of patients with $\mathrm{AF}$ and $85 \%$ of patients with DM. HT and DM have been identified as comorbid diseases that cause more intracranial hemorrhage. In this respect, we think that strict blood glucose and blood pressure monitoring are important.

The cause of death of 2 patients was secondary infections regardless of the procedure. We consider that the cause of death of the other 3 patients was difficult endovascular procedure due to poor vascular structure.

Success in the treatment of acute stroke is often timedependent. Many studies have shown that symptom-needle duration is an important factor in clinical recovery $[9,10]$. In our study, onset-to-gate and gate-to-puncture durations were found longer than the recommended times, not statistically significant. But we think that this problem can be partially overcome by shortening the transfer durations.

\section{Conclusion}

The fact that the rate of intracranial bleeding was higher in patients who received bolus or full dose IV-tPA compared to patients who had only MT, showed that we should make a more careful patient selection for IV-tPA.

\section{References}

1. Higashida RT, Furlan AJ, Roberts H, Tomsick T, Connors B, et-al. Trial design and reporting standards for intra-arterial cerebral thrombolysis for acute ischemic stroke. Stroke. 2003; 34: e109-137. PubMed: https://pubmed.ncbi.nlm.nih.gov/12869717/

2. NINDS rt-PA Stroke Study Group. Tissue plasminogen activator for acute ischemic stroke. N Engl J Med 1995; 333: 1581-1587.

PubMed: https://pubmed.ncbi.nlm.nih.gov/7477192/

3. Hache W, Kaste M, Bluhmki E, Brozman M, Dávalos A, et al. ECASS investigators. Thrombolysis with alteplase 3 to 4.5 hours after acute ischemic stroke. N Engl J Med. 2008; 359: 1317-1329.

PubMed: https://pubmed.ncbi.nlm.nih.gov/18815396/

4. Powers WJ, Derdeny CP, Biller J, Coffey CS, Hoh BL, et al. American Heart Association Stroke Council. 2015 American Heart Association/ American Stroke Association Focused Update of the 2013 Guidelines fort he Early Management of Patients With Acute Ischemic Stroke Regarding Endovascular Treatment: A Guideline for Healthcare Professionals From the American Heart Association/American Stroke Association. Stroke 2015; 46: 3020-3035.

PubMed: https://pubmed.ncbi.nlm.nih.gov/26123479/ 
5. Hacke W. A new DAWN for imaging-based selection in the treat- ment of acute stroke. N Engl J Med. 2018; 378: 81-83.

PubMed: https://pubmed.ncbi.nlm.nih.gov/29129127/

6. Albers GW, Marks MP, Kemp S, Christensen S, Tsai JP, et al. Thrombectomy for stroke at 6 to 16 hours with selection by perfusion imaging. N Engl J Med. 2018; 378: 708-718.

PubMed: https://pubmed.ncbi.nlm.nih.gov/29364767/

7. Goyal M, Demchuk AM, Menon BK, Eesa M, Rempel JL, et al Randomized assessment of rapid endovascular treatment of ischemic stroke. N Engl J Med. 372: 1019-1030.

PubMed: https://pubmed.ncbi.nlm.nih.gov/25671798/
8. Gory B, Eldesouky I, Sivan-Hoffmann R, Rabilloud M, Ong E, et al. Outcomes of stent retriever thrombectomy in basilar artery occlusion: an observational study and systematic review. J Neurol Neurosurg Psychiatry. 2016; 87: 520-525.

PubMed: https://pubmed.ncbi.nlm.nih.gov/25986363/

9. Wardlaw JM, Murray V, Berge E, Del Zoppo GJ. Thrombolysis for acute ischaemic stroke. Cochrane Database Syst Rev. 2009; 4: Cd000213. PubMed: https://pubmed.ncbi.nlm.nih.gov/25072528/

10. Azzimondi G, Bassein L, Fiorani L, Nonino F, Montaguti U, et al. Variables associated with hospital arrival time after stroke: effect of delay on the clinical efficiency of early treatment. Stroke. 1997; 28: 537-542. PubMed: https://pubmed.ncbi.nlm.nih.gov/9056608/ 